\title{
LA IDEA FEMINISTA DE LA POLÍTICA DESDE EL SUR GLOBAL
}

\author{
Breno Henrique Ferreira Cypriano \\ Universidade Federal de Minas Gerais
}

\begin{abstract}
Resumen: Este artículo discute la importancia y centralidad de los conocimientos acerca de "la política" y "lo político" desde la perspectiva feminista desde el Sur global. Para eso, es necesario una abordaje de las principales discusiones feministas conceptuales y un intento de diseño de un panorama pos(de)colonial del concepto de "Ia" política.
\end{abstract}

Palabras clave: teoría política feminista; política; pensamiento político latinoamericano; pensamiento poscolonial.

Después de un agotador traslado y trabajo de deconstrucción, se puede decir que en las últimas décadas el feminismo inició una nueva etapa: un construir teórico que es fielmente feminista, centrando principalmente en las discusiones sobre el conocimiento de "la política" y de "lo político".' Esto quiere decir que el feminismo académico comenzó a alejarse de la actividad crítica estrictamente conceptual a una formativa, que fundamentada en las teorías filosófica, teórica y científica dio forma a una teoría. ${ }^{2}$ Desde la perspectiva de esta teoría llamada "teoría política feminista", se empieza a preguntar acerca de lo que ella misma representa epistémicamente para los conocimientos especializados sobre lo que es "lo político" y lo que es "la política", como también se debe entender básicamente lo que representa el campo propio de conocimiento político. Si así debe ser, para la localización y la contextualización de esta agenda teórica y de investigación, debería asociarla con la importancia relativa asumida por los debates sobre la modernidad, como sus consecuencias y repercusiones en la densificación de las críticas paradigmáticas posmodernas y pos-estructuralistas acerca de los discursos de las

Copyright (C) 2014 by Revista Estudos Feministas.

1 La diferenciación entre "la política" y "lo político" es discutida por Claude Lefort (1991 [1986]). Para él "la política" es un objeto de estudio de la ciencia política y de la sociología política, que es construido o delimitado desde un hecho político, o sea, un hecho particular que es distinto de los demás hechos sociales. Para este autor, la definición del concepto de "la política" proviene de una observación o construcción epistémica que no deriva de una experiencia de la vida social. La concepción de "lo político" dice de la constitución de la sociedad, ya que hace visible el proceso crítico por lo cual una sociedad va a ser ordenada y unificada. "Lo político" es entonces un modo de institución social que dice acerca de un "lugar de poder". Otras discusiones sobre esta diferencia están en Chantal MOUFFE, 2005; y Pierre ROSANVALLON, 2003.

2 Judith GRANT, 1993. 
ausencias, así como la reflexión sobre los límites de las cadenas recientes del liberalismo, de la colonialidad, del patriarcado y del imperialismo.

En las últimas dos décadas, una de las discusiones más controvertidas dentro de la teoría política feminista fue aquella sobre la representación política feminista, y estuvo centrada en una paradoja: ¿sería la política de las ideas o la política de la presencia la cuestión central para ser tratada en las discusiones acerca de mujer y política? ${ }^{3}$ Más que una cuestión cuantitativa o cualitativa, muchos debates y artículos se produjeron en torno de la cuestión de las cuotas (o cupos) para mujeres en los partidos políticos y en los parlamentos y cámaras de diputados. Esa fue una tendencia mundial, que generó una extensiva formación de redes de estudios y centros de capacitación para mujeres. Las mujeres deberían estar "representadas", ésta era una normativa que empezaba a ser difundida por muchas organizaciones internacionales. La historia real de la adopción de dichos cupos nos remonta a Eva Perón y al Partido Peronista en 1948, cuando ella acordó con el Consejo Superior Peronista un cupo para mujeres en la primera elección en la que las mujeres argentinas pudieran votar y ser votadas ${ }^{4}$. Distinto de todos los trabajos que resaltan las acciones afirmativas nórdicas como las primeras en el mundo, la experiencia pionera argentina liderada por Eva Perón, aunque no feminista, produjo consecuencias a posteriori con la conquista de los cupos constitucionales. Esto debido a una lucha de los movimientos feministas. Este ejemplo argentino tan claro de la agenda de lucha y fuerza feminista latinoamericana en la política, se nos plantea los interrogantes ipor qué no serían esos mecanismos como cupos o cuotas ideales para la conquista de los deseos ideológicos feministas?, y si pudiéramos ser un poco más propositivos, ¿cuáles serían las posibles formas de pensarse "la política" y "lo político"?

\section{Desde la contribución feminista latinoamericana: logros y desafíos}

Visto lo que ya se ha presentado, puedo responder a la pregunta: ¿Por qué el enfoque en la América Latina y en el Sur Global? Porque esta es una región geográfica donde el sexismo, el patriarcado y la cultura religiosa heredada se dibujan históricamente hasta nuestros días, y mantienen sus influencias sobre el pensamiento político y social. A pesar de este modelo, aún colonial, una reacción en cadena en todo el continente permitió la resistencia de las minorías políticas y sociales, lo que resultó en la aparición y el establecimiento de los movimientos feministas y de mujeres. La condición política, cultural y social se acercó entre los países del continente y, por lo tanto, permitió algunas generalizaciones. Empero no nos olvidemos las diversidades y diferencias presentes en cada país y región del continente. Sería aquí importante utilizar la idea de "Tercer feminismo" propuesto por Karina Bidaseca sobre el movimiento feminista en el Sur desde las epistemologías de fronteras:

El Tercer Feminismo que propongo pensar es aquél que logrando interpelar a la sociedad toda sobre las violencias contra el género, se inscriba en una genealogía de la memoria poscolonial, que necesita recuperar una memoria epistémica, que no omita las contribuciones del feminismo chicano (el sur del norte) pero que se sitúe en nuestro Sur.

Julieta Kirkwood ${ }^{6}$ establece que el feminismo en América Latina puede ser visto a través de los "nudos" políticos feministas, conjunciones que surgieran desde las reuniones

\footnotetext{
${ }^{3}$ Anne PHILLIPS, 1995.

4 Carolina BARRY, 2011.

${ }^{5}$ Karina BIDASECA, 2012 , p. 42.

${ }^{6}$ Julieta KIRKWOOD, 2010 [1986].
} 
feministas en el continente y que es sin duda un logro del espacio político para las mujeres a través del diálogo entre las activistas feministas y las mujeres políticas (y, más allá, podríamos añadir el feminismo académico, ya que esta forma se relaciona y corteja con las prácticas de los dos campos). Los nudos feministas, como apuntados por Kirkwood, están y estarán presentes cuando surgen interacciones entre distintas facciones de mujeres, entre feministas y políticas, entre diferentes encuentros, que aún están presentes y que persisten en el tiempo. Esto puede ser deshecho con saber y poder. La cuestión es que no hay consenso entre la noción de qué es la feminidad, y de qué es ser mujer para la agenda de esos grupos, y cómo eso se traduce en la política y en las políticas públicas ${ }^{7}$. Estudios sobre las acciones afirmativas y la representación de las mujeres fueron realizados por Alejandra Castillo ${ }^{8}$ que, al pensar el feminismo como una política "de lo múltiple", según ella, dos tipos de acción serían posibles: las políticas de la acción afirmativa y las políticas de la interrupción. Las políticas de acción afirmativa son aquellas que, como dicho anteriormente, fueron tratadas como la idea de la política de la presencia, lo que enfatizó un concepto de una "mujer" femenina y cuidadora, portadora de una esencia de cuidado; por otro lado hay las políticas de la interrupción, donde las mujeres proponen un cuestionamiento al modo moderno de la política, como lo hicieron Gloria Anzaldúa ${ }^{9} y$ Julieta Kirkwood, ${ }^{10}$ ya que hasta ahora todo lo que se hizo fue poco. Como señala Castillo,

No se puede ser feminista sólo habitando en los márgenes: habitando la tranquilidad del margen de la historia (en la escritura de la 'otra historia'); habitando en los márgenes de la lengua (en la audacia de la creación de otras hablas, casi siempre de los sentimientos); habitando, por último, en los márgenes del poder (en la creencia de políticas de la diferencia).

Castillo demostró que las políticas de la interrupción deben ser un juego complejo entre lo universal y lo particular, de las márgenes hacia el centro, y como la autora retoma la idea de los nudos de Kirkwood, hay que reinventar la políica como un todo, y por eso no puede ser una política de interés, sino un proyecto de transformación total. De este modo, el feminismo trabajará en una zona fronteriza del pensamiento haciendo la traducción del pensamiento político, reemplazado las grandes teorías, y sospechando de los universales. Y aún más, como argumenta Cláudia Lima Costa, ${ }^{12}$ percibimos el "tráfico internacional de conceptos", que ha enflaquecido sustancialmente las relaciones entre las teorías y los lugares.

\section{La ontologización del concepto de "la política": política como traducción}

Para una discusión general sobre la noción de lo que es "la política", la teoría social y política contemporánea pasa de una concepción limitada de Weber ${ }^{13}$ de "la política como dominación" - entendida como los múltiples esfuerzos realizados para la participación en el poder o para influir en su división entre los Estados o en un solo Estado - a una visión más grande y potencialmente emancipadora, la concepción arendtiana de "la política como la libertad", que culminó en la esfera política y en el interés público del nuevo, centrado en la diversidad humana, y por lo tanto es una acción participativa en la vida

\footnotetext{
7 Laura MASSON, 2007

${ }^{8}$ Alejandra CASTILLO, 2011.

9 Gloria ANZALDÚA, 1987

${ }^{10}$ KIRKWOOD, 2010 [1986]

11 CASTILLO, 2011 , p. 23.

12 Cláudia Lima COSTA, 2011.
} 
pública. Es cierto que los enfoques feministas no siguen muy adelante con estos debates. Se podría decir que el concepto más avanzado en intentos políticamente orquestados por las feministas fue el patriarcado, ya que proporcionó las herramientas analíticas para comprender la estabilización, y también para repensar las jerarquías existentes entre los sexos en las relaciones políticas.

Judith Butler ${ }^{14}$ es una teórica feminista presente en las discusiones más densas sobre "la política". Ella produjo una interpretación radical de la democracia y sobre la idea de una lucha de la "futuridad" en intervenir en el rol de lo teórico. Eso sería unirse a esta lucha tanto para dar forma a "la política", así como constituir "lo político". De acuerdo con Butler, los límites entre lo óntico y ontológico serían borrosos, o sea: "la política" se confundió con "lo político" (†odas las veces que nos referimos a óntico y ontológico será a las categorías heideggerianas)..$^{15}$ Para Samuel Chambers y Terrell Carver, ${ }^{16}$ la "política problematizada" (troubling politics) se refiere a una variedad de encuentros de Judith Butler con "Ia política", con "Io político" y con la teoría política, que pueden ser comprendidos a través de tres niveles vertebradores: (i) la noción de que nunca podrá haber un sujeto prontamente fabricado, ya que este sujeto debe tener como reto las perturbaciones y poner en cuestión, de manera consistente, sus propias condiciones; (ii) la noción y motivación transformativas a cerca de las concepciones de la política, y también una recusa a una noción estática de la política, ya que por intermedio de una visión radical se puede dejar en abierto la busca por el futuro de la democracia; y (iii) la noción de que es necesaria la problematización pulverizada de una teoría, o sea, deberían estar problematizadas las cuestiones de adentro, de afuera, lo que correspondería a una teoría no-linear. De este modo, la teoría butleriana busca una concepción de política que envuelva tanto la dimensión teorética, como la dimensión práctica.

Ernesto Laclau y Chantal Mouffe ${ }^{17}$ sostienen que un cambio en el contenido óntico daría lugar a nuevos paradigmas ontológicos, es decir, la redefinición de la política conduciría a otros paradigmas del concepto de "lo político". El concepto de Carl Schmitt ${ }^{18}$ en el antagonismo, basado en el eje de las controversias entre amigo y enemigo, y también la noción de consenso político deliberativo por John Rawls ${ }^{19}$ y Jürgen Habermas, ${ }^{20}$ han sido desafiados por nociones tales como las de Mouffe. ${ }^{21}$ Ésta prefiere destacar el "pluralismo agonístico", en el cual el eje es una disputa entre amigos. Agregando a este desplazamiento, se puede decir que la noción óntica de la política como dominación reemplazada por la idea de la libertad política confluiría con el cambio paradigmático de pensar "lo político" como antagonismo con la idea de consenso o agonismo. Es precisamente la sensibilidad feminista y la percepción de "lo político" que también hizo que estos elementos de transición convergieran en un nuevo concepto de lo "público", esta vez ampliado y renovado, así como la percepción de los conceptos del privado y del personal, interpelados ya como inherentemente politizados (como la familia, la reproducción, el cuidado, el cuerpo), contribuyen a la superación de las relaciones de poder desiguales de género. La disputa entre los movimientos feministas de América Latina por la noción de lo político, o considerado

\footnotetext{
${ }^{13}$ Max WEBER, 2004 [1919].

14 Judith P. BUTLER, 1993; BUTLER; LACLAU y ZIZEK, 2000.

${ }^{15}$ Martin HEIDEGGER, 2009 [1927]; y MOUFFE, 2006

${ }^{16}$ CHAMBERS Y CARVER, 2008.

17 Ernesto LACLAU y Chantal MOUFFE, 2004 [1985].

${ }^{18}$ Carl SCHMITT, 1994 [1927].

19 John RAWLS, 2000 [1995].

20 Jürgen HABERMAS, 1987 [1981].

${ }^{21}$ MOUFFE, 1996 [1993].
} 
como antagonismo o agonismo, también sirve para pensar y reflejar este cambio de paradigma.

A partir de la relevancia de la teoría generada a partir del "tercer mundo", las críticas a las nuevas escalas de las interacciones políticas producidas por la globalización a partir de un país (o grupo de países) de los países del Sur, hizo posible el poder "hablar dentro de" las narrativas hegemónicas y, sin embargo, proponer un proyecto que no sólo se base en el diálogo Sur con el Norte, pero sobre todo con las diferencias y diversidades infinitas en el Sur. Eso hace crear un diálogo con la producción existente en el campo de conocimiento político dominante, sino también con las diversas perspectivas incluidas en lo que podría denominarse una "teoría política feminista" occidental sumadas a las contribuciones del pensamiento político feminista latinoamericano. Así, dice Diana Maffia, ${ }^{22}$ "las mujeres invitamos a repensar el lenguaje, a invertir con nuevas energías [...] Y tenemos algo más para traccionar estos cambios plurales, una intransigencia semántica: sólo llamaremos 'democracia' a un sistema capaz de desnaturalizar todas las formas de hegemonía y subordinación.", por lo tanto, la contribución que cualquier análisis feminista se pone a pensar está no sólo en la política del Estado, o en la política representativa, pero en "la política" o en "lo político" como una manera de inclusión/traducción en diversas esferas de la vida, de la manera más amplia posible (Fig. 1).

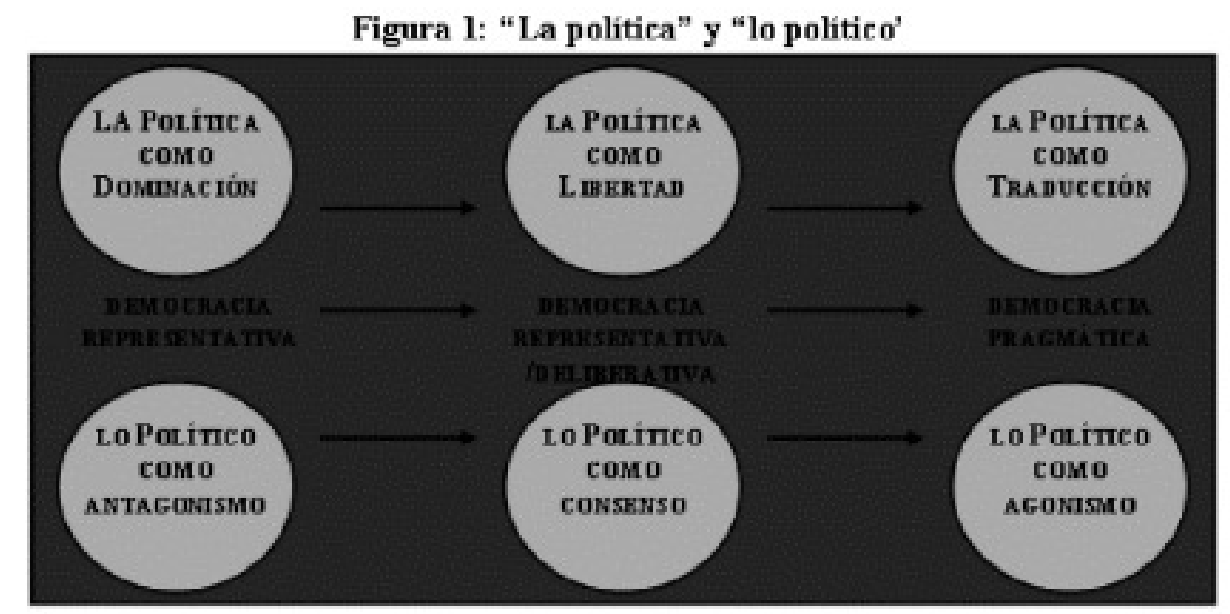

Boaventura de Sousa Santos ${ }^{23}$ afirma que el trabajo de traducción se centrará en el conocimiento y las prácticas de los sujetos políicos, que a su vez permitan una inteligibilidad recíproca entre ambas las esferas. Sonia Alvarez señala que la traducción es un proceso de apertura para el otro y él sería en sí "político y teóricamente esencial para forjar alianzas políticas y epistemologías feministas, antirracistas y poscoloniales/pos-occidentales [...]". ${ }^{24} \mathrm{~A}$ esta noción de la "política como traducción", que convergen con la experimentación de una nueva fase u ola del movimiento feminista como algunas autoras destacan, ${ }^{25}$ hay que prestar atención a dos direcciones posibles del proceso de traducción: una aproximación de los

${ }^{22}$ Diana MAFFÍA, 2003, p. 76.

${ }^{23}$ Boaventura de Sousa SANTOS, 2007

${ }^{24}$ Sonia E. ALVAREZ, 2009, p. 743, nuestra traducción.

${ }^{25}$ Marlise MATOS, 2010. 
movimientos con la academia y de la academia/movimientos con el Estado, lo que permite una agenda con intereses públicos compartidos. Por lo tanto, se cree que esto sería una norma invocada por aquellos hombres y mujeres que buscan profundizar y ampliar las prácticas democráticas, en el supuesto de que las experiencias de exclusión, opresión y marginación lleven a las demandas por inclusión. ${ }^{26}$ Se podría decir que el redimensionamiento de la noción de justicia, incluyendo ahora una dimensión política, hace reinvenciones de las asociaciones de género en el ámbito doméstico (la división del trabajo doméstico, con la inclusión de los hombres en estas actividades), como en otras relaciones laborales en el sector privado y en el ámbito público, tanto en sectores estatales y no estatales. Bajo la noción de representación y la política ordinaria simbólica, las prácticas políticas convencionales deben ahora asumir la responsabilidad de las prácticas de exclusión internas a sí mismas ${ }^{27}$.

Aunque posiblemente la indigencia de un concepto de "la política" sea, quizás, una necesidad teórica hegemónica, el papel feminista del conocimiento para este caso sería estrictamente de desestabilización. La búsqueda de la politización de ciertos conceptos, flexionando lo que sería "lo político", fue y es una estrategia contra-hegemónica y que la multiplicidad de significados de "la políica" sería lo que caracterizaría la radicalidad del feminismo. Sin embargo, el movimiento político en la academia y el activismo político, para adentrar en los espacios del Estado y por exigir que "lo personal es político", ha demostrado la necesidad de pensar en un parámetro común en lo que sería "la política" a través de una estabilización/estabilidad teórica que es crítica, emancipadora y pragmática.

La desestabilización paradigmática del nivel óntico - principalmente el impacto del concepto político de Arendt - a través de la pregunta de lo que es "lo político" causó mucha inestabilidad teorética, por eso el enfoque feminista se preguntó acerca de los paradigmas ontológicos. A diferencia de la noción de Laclau y Mouffe,$^{28}$ la teoría feminista caminó contra la corriente: propuso nuevos paradigmas ontológicos, y que han producido ahora una nueva definición de "la política" - que contendría una visión que incluye una mirada micro institucional acerca de la pulverización del locus y de consideraciones anti-patriarcales, anti-maculinistas, y rechazando los binarismos, una concepción anti-dicotómica, y que aún sigue considerando la dinámica de la transnacionalización y del agonismo.

"La política" como traducción desde una perspectiva pos(de)colonial va a buscar transponer las barreras y las fronteras construidas en el campo del conocimiento político comenzando por medio de desestabilizaciones, y después implicando en la conformación de un "otro" campo que tenga su propia discusión epistemológica, ontológica, metodológica, estético-discursiva y también que goce de sus propios conceptos centrales. Por eso, una mirada feminista, y también una mirada desde el Sur global, tendrán que cuestionar siempre o que se entiende como "la política". Si es verdad que los movimientos feministas y de mujeres contribuyen y construyen relaciones (demandas y respuestas) para los espacios académicos y espacios institucionales/políticos (como el Estado), es razonable suponer que estas demandas y respuestas (re)crean nuevos campos de interlocución epistémica/teórica (y más, prácticas diferenciadas), como un nuevo campo feminista crítico de género o un "campo científico-crítico-emancipatório de las diferencias como experiencia de la descolonización académica"29. Para Marlise Matos, este campo "es responsable por multiplicar los lugares desde los cuales debe ser ejercitada la política, el poder y la democracia, sobre todo para aquellos grupos que están deseosos de reinventar esa democracia y también la justicia social".$^{30}$ Si los movimientos de mujeres y

\footnotetext{
${ }^{26}$ Iris Marion YOUNG, 2000a.

27 FRASER, 2005.

${ }^{28}$ LACLAU y MOUFFE, 2004 [1985]

${ }^{29}$ MATOS, 2012

${ }^{30}$ MATOS, 2012, p. 92, nuestra traducción, itálicos de la autora.
} 
feministas (las activistas) son capaces en la interlocución con el feminismo académico y también, como señala Kirkwood, con las "políticas" (o burócratas), creando lo "nudo feminista", entonces es importante suponer que sea necesario (y urgente) la delimitación de una teoría política feminista o un pensamiento crítico/emancipatrio feminista de "la política" y de "lo político" que reposicione y reintroduzca los grandes temas o demandas en una nueva episteme política.

Si una nueva episteme política y un nuevo campo de conocimiento político serán posibles, es necesario una apuesta de si pensar "la política" como traducción. Traducción aquí significa la busca por conocimiento de la experiencia que ocurre a través de las "zonas de contactos" múltiples, que son zonas de frontera producidas como una alternativa a una teoría general. ${ }^{31}$ Así hay in intento de superar un único universal teniendo como último fin la multiplicación de los universales que se crean en las diversas zonas de contactos. La relación y conexión entre los saberes científicos y los saberes filosóficos, sumadas a los saberes de la calle, aportan para la composición de este "otro" campo, u otros, donde los conceptos centrales de estos distintos discursos convergirían para la producción de un lenguaje especializado, con un contenido significante propio, sui generis, que puede ser compartido, publicitado y puesto en discusión, donde los conocimientos corpóreos, regionalizados, propios, vividos, pos(de)colonizados, surge por intermedio de las "experiencias", los "puntos de vista", ${ }^{32}$ los "encuentros de saberes". ${ }^{33}$

La demanda por un campo de conocimiento que traduzca las experiencias desde el Sur global (en especial América Latina), en este caso desde dónde hablamos, sugiere que no se traduzca solamente lo que se ha hecho en Estados Unidos o en Europa, o sea, en el Norte global, y por lo tanto que se va más allá de lo que se conoce hoy como "teoría política feminista", un grupo cerrado de auto-citación compuesto por pocas/pocos teóricas/ teóricos, que de acuerdo con Susan Okin ${ }^{34}$ fue instituido por una especie de "carta de fundación" por la cual prácticamente se puede prever un destino glorioso o, quizás, un fin desastroso de esta "hermandad". Dos estrategias ahí son importantes: la recusa al tráfico de teorías y saberes, o un uso crítico de ellas, una forma de "perturbar el texto colonial", ya que, según Bidaseca, "[el] conocimiento occidental está colonizado. Se trata de des- colonizarlo o incluir otras formas de generar conocimiento." 35

Así, la dimensión participativa y/o deliberativa de los movimientos sociales en América Latina, como también la inclusión de voces feministas en los Estados latinoamericanos, son importantes dados empíricos de cambios reales en la política. Cuestiones como cupos y cuotas son importantes ya que ayudan en transformaciones simbólicas, sociales y políticas, aunque no sean la única o mejor salida para los problemas que llevan la ausencia de las mujeres en la política. Hay que tener en cuenta no solo la representación política formal, sino todo que es "lo político", desde la lucha feminista y de las mujeres, hasta las presencias de mujeres en el poder. Sí el feminismo ontológicamente cambia la concepción de la dimensión del poder, o sea, un cambio de que es "lo político", la representación y la legitimación de este poder va cambiar: desde la propia idealización hasta la instancia do que llamamos de "la política". En suma, las articulaciones y los contactos entre diversas experiencias y encuentros en distintas esferas por los feminismos, lleva a creer en una nueva idea de la política desde el Sur global. Y que así sea.

\footnotetext{
31 SANTOS, 2007.

${ }^{32}$ Para una discusión de esos conceptos en la teoría feminista y la visión del pensamiento feminista latinoamericano conferir CYPRIANO, 2013.

33 SANTOS, 2007; Walter MIGNOLO, 2010.

${ }^{34}$ Susan Moller OKIN, 1992

35 BIDASECA, 2010, p. 20, itálicos de la autora.
} 


\section{Referencias}

ALVAREZ, Sonia E. "Construindo uma política feminista translocal da tradução". Revista Estudos Feministas, Florianópolis, v. 17, n. 3, p. 743-753, set./dez. 2009.

ANZALDÚA, Gloria. Bordelands/La frontera. San Francisco, CA: Aunt Lute, 1987.

BARRY, Carolina. Eva Perón y la organización política de las mujeres. Documento de trabajo. Buenos Aires: UCEMA, 2011.

BIDASECA, Karina. Pertubando el texto colonial: los estudios (pos)coloniales en América Latina. Buenos Aires: SB, 2010.

"Primeras exhalaciones: políticas de la memoria, genealogias coloniales y 'tercer feminismo'”. Papeles de Trabajo, Buenos Aires, año 6, n. 10, p. 30-45, 2012.

BUTLER, Judith P. Bodies that matter: on the discursive limits of "sex". New York and London: Routledge, 1993.

BUTLER, Judith P.; LACLAU, Ernesto; •l•EK, Slavoj. Contingency, hegemony, universality: contemporary dialogues on the left. London and New York: Verso, 2000.

CASTILLO, Alejandra. Nudos feministas. Santiago: Palinodia, 2011.

CHAMBERS, Samuel Allen; CARVER, Terrell. Judith Butler and political theory: troubling politics. New York: Routledge, 2008.

COSTA, Cláudia Lima. "As teorias feministas nas Américas e a política transnacional da tradução". Revista Estudos Feministas, Florianópolis, v. 8, n. 2, p. 43-49, 2000.

CYPRIANO, Breno. "Construções do pensamento feminista latino-americano". Revista Estudos Feministas, Florianópolis, v. 21, n.1, p. 11-39, jan./abr. 2013.

DWORKIN, Ronald. Uma questão de princípio. Traducción de Luís Carlos Borges. São Paulo, SP: Martins Fontes, 2000 [1985].

FRASER, Nancy. Reframing justice. Amsterdam: Royal Van Gorcum, 2005.

GRANT, Judith. Fundamental feminism: contesting the core concepts of feminist theory. London: Routledge, 1993.

HABERMAS, Jürgen. Teoria de la acción comunicativa. Traducción de Manuel Jimenez Redondo. 2. v. Madrid: Taurus, 1987 [1981].

HEIDEGGER, Martin. Ser e tempo. Traducción de Márcia Sá Cavalcante Schuback. 4. ed. Petrópolis: Vozes, 2009 [1927].

HONNETH, Axel. Luta por reconhecimento: a gramática moral dos conflitos sociais. Traducción de Luiz Repa. São Paulo: Editora 34, 2003 [1992].

KIRKWOOD, Julieta. Ser política en Chile: las feministas y los partidos. Santiago: LOM Ediciones, 2010 [1986].

LACLAU, Ernesto; MOUFFE, Chantal. Hegemonía y estrategia socialista: hacia una radicalización de la democracia. Traducción de Ernesto Laclau. Buenos Aires, ARG: Fondo de Cultura Económica, 2004 [1985].

LEFORT, Claude. Pensando o político: ensaios sobre a democracia, revolução e liberdade. Tradução de Eliana M. Souza. Rio de Janeiro, RJ: Paz e Terra, 1991 [1986].

MAFFIA, Diana. Socialismo y liberalismo en la teoría política contemporánea. In: BORON, Atílio (Comp.). Filosofía política contemporánea. Buenos Aires: Consejo Latinoamericano de Ciencias Sociales, 2004. p. 173-177.

MASSON, Laura. Feministas en todas partes: una etnografía de espacios y narrativas feministas en Argentina. Buenos Aires: Prometeo Libros, 2007.

MATOS, Marlise. Movimento e teoria feminista: possível reconstruir a teoria feminista a partir do sul global? Revista Sociologia e Política, Curitiba, v. 18, n. 36, p. 67-92, 2010.

634 Estudos Feministas, Florianópolis, 22(2): 627-635, maio-agosto/2014 
O campo científico-crítico-emancipatório das diferenças como experiência de descolonização acadêmica. In: BIROLI, Flávia; MIGUEL, Luis Felipe (Eds.). Teoria política e feminismo: abordagens brasileiras. Vinhedo: Editora Horizonte, 2012. p. 47-102.

MIGNOLO, Walter. Desobediencia epistémica: retórica de la modernidad, lógica de la colonialidad y gramatica de la descolonialidad. Buenos Aires: Ediciones del signo, 2010.

MOUFFE, Chantal. O regresso do político. Traducción de Ana Simões. Lisboa: Gradiva, 1996 [1993].

. On the political. London and New York: Routledge, 2005.

NUSSBAUM, Martha. Women and human development: the capabilities approach. New York: Cambridge University Press, 2000.

OKIN, Susan Moller. Justice, gender and the family. New York: Basic Books, 1989. "Afterword to the 1992 edition." In: OKIN, S. M. Women in western political thought. 2nd. ed. Princeton: Princeton University Press, 1992. p. 309-340.

PHILLIPS, Anne. The politics of presence: the political representation of gender, ethnicity, and race. Oxford and New York: Oxford University Press, 1995.

RAWLS, John. O liberalismo político. Traducción de Dinah de Abreu Azevedo. 2. ed. São Paulo: Editora Ática, 2000 [1995].

ROSANVALLON, Pierre. Pour une histoire conceptuelle du politique. Paris: Seuil, 2003.

SANTOS, Boaventura de Sousa. Renovar a teoria crítica e reinventar a emancipação social. São Paulo, SP: Boitempo, 2007.

SCHMITT, Carl. "The concept of the political". In: KAES, Anton; JAY, Martin; DIMENDBERG, Edward (Eds.), The Weimar Republic sourcebook. Berkeley and Los Angeles: University of California Press, 1994 [1927]. p. 342-344.

SEN, Amartya K. Desigualdade reexaminada. Traducción de Ricardo Doninelli Mendes. Rio de Janeiro: Editora Record, 2001 [1992].

TAYLOR, Charles. El multiculturalismo y "la política del reconocimiento". Traducción de Mónica Utrilla de Neira. Ciudad de México: Fondo de Cultura Económica, 1993 [1992].

WALZER, Michael. Esferas da justiça: uma defesa do pluralismo e da igualdade. Tradução Jussara Simões. São Paulo: Martins Fontes, 2003 [1983].

WEBER, Max. "A política como vocação". In: WEBER, M. Ciência e política: duas vocações. Traducción de Leônidas Hegemberg y Octany Silveira da Mota. 15. ed. São Paulo: Cultrix, 2004 [1919]. p. 55-124.

YOUNG, Iris Marion. Inclusion and democracy. Oxford: Oxford University Press, 2000a. La justicia y la política de la diferencia. Traducción de Silvina Álvarez. Madríd: Ediciones Cátedra, 2000b [1990].

[Recebido em fevereiro de 2014 e aceito para publicação em abril de 2014.

The Feminist Idea of Politics from the Global South Perspective

Abstract: This article seeks to discuss the importance and centrality of the knowledge about "politics" and "the political" from a feminist perspective from the Global South. For this purpose, it's necessary to approach the main conceptual feminist discussions, and attempt to design a post(de)colonial scenario of the actual concept of "politics".

Key Words: Feminist Political Theory; Politics; Latin American Political Thought; Postcolonial Thought. 